\title{
La percepción social hacia las personas con síndrome de Down: la escala EPSD-1
}

\author{
Jesús Molina Saorín \\ Universidad de Murcia, España \\ Rui Manuel Nunes Corredeira \\ Universidad de Porto, Portugal \\ Mónica Vallejo Ruiz \\ Universidad de Murcia, España
}

\section{Resumen}

En esta investigación, financiada por varias entidades, hemos diseñado un instrumento que permite conocer la percepción que tienen los estudiantes universitarios de Educación Física sobre las personas con síndrome de Down. Al instrumento lo hemos denominado escala de Pecepción Social hacia las personas con síndrome de Down EPSD1 , y en su diseño se recogen importantes variables psicosociales, al tiempo que se busca dar cuenta de sus propiedades para aplicarla a otros contextos. Tras un análisis factorial, ofrecemos diez grandes factores que abordan temas como la exclusión social de las personas con síndrome de Down, su autonomía e independencia, sus relaciones afectivo-sexuales, su aceptación social y educativa, su integración, la actitud familiar, la formación docente y el proteccionismo social hacia estas personas. La muestra inicial la componen 1.796 participantes y los resultados indican que se trata de un instrumento fiable y válido para su aplicación. Esta escala resulta de gran utilidad en el ámbito de las Ciencias Sociales, ofreciendo una relación latente con respecto a la formación inicial que, desde la universidad, se ofrece a los estudiantes universitarios. Mediante una metodología cuantitativa, los resultados muestran que sus propiedades psicométricas son altamente satisfactorias, motivo por el cual sugerimos realizar nuevos estudios longitudinales y transversales a partir de su uso aplicado a diferentes poblaciones, con objeto de profundizar sobre la percepción social hacia las personas con síndrome de Down, aportando datos sobre la tendencia y evolución al respecto en los últimos años.

\section{Palabras clave}

Contacto:
Validación - análisis factorial - síndrome de Down - percepción social. 


\section{The social perception of people with Down syndrome: the EPSD-1 scale}

Jesús Molina Saorín

Universidad de Murcia, España

Rui Manuel Nunes Corredeira

Universidad de Porto, Portugal

Mónica Vallejo Ruiz

Universidad de Murcia, España

Contact:

Jesús Molina Saorín

Universidad de Murcia

Campus Universitario de Espinardo,

Facultad de Educación,

Espinardo (Murcia)

jesusmol@um.es

\begin{abstract}
In this research, which was financed by several institutions, we have designed an instrument that allows us to know the perception that university students of Physical Education have of people with Down syndrome. We have called this instrument the scale of Social Perception of people with Down syndrome EPSD-1, and in its design important psychosocial variables are recognized, whilst trying to discuss its properties in order to apply it to other contexts. Based on an analysis of factors, we offer ten main factors that deal with issues such as the social exclusion of people with Down syndrome, their autonomy and independence, their affective-sexual relations, their social and educational acceptance, their integration, their family attitudes, teacher education, and the social protectionism of these people. The initial sample is composed of 1.796 participants, and the results indicate that the scale devised here is a reliable and valid instrument in its application. This scale can be of great value for the Social Sciences, offering a latent relation with respect to the initial education which, since the university, is offered to students of higher education. Using a quantitative methodology, the results show that the psychometric properties of the scale are highly satisfactory, prompting us to suggest the conduction of new longitudinal and transversal studies using it to different populations with the purpose of furthering our knowledge of the social perception of people with Down syndrome, and of bringing out new facts about the tendency and evolution of these theme during the last years.
\end{abstract}

\section{Keywords}

Validation - Analysis of factors - Down's syndrome - Social perception. 
Intentar resumir la evolución social de la discapacidad intelectual, atravesando sus diferentes etapas históricas, resultaría prácticamente imposible en este trabajo porque nos encontramos frente a un tema controvertido y complejo en su esencia misma, que además ha sufrido innumerables cambios (RIEF; HEIMBURGE, 2000; CÁCERES, 2004; ILLÁN, 2001; GRANIZO; NAYLOR; DEL BARRIO, 2006). Durante los últimos años, la sociedad ha dado pasos importantes en su concepción acerca de la discapacidad, en general, y del síndrome de Down (SD) en particular (MOYA, 1999). Hemos ido superando viejos prejuicios que estereotipan y califican a quienes tienen SD, tildándoles de menos capaces (EBBECK; WEISS, 1998). Con el paso de los años, vamos incorporando la idea de que todas las personas somos iguales, independientemente de que tengamos discapacidad o no, del sexo con el que nazcamos, de nuestra condición social, orientación sexual, religión, etnia, etcétera (VERDUG0, 2002). Todos los avances sociales han tenido su reflejo en el lenguaje, siendo la sociedad que lo utiliza quien describe las especificidades de cada grupo social y muestra sus concepciones en cada momento. Sin embargo, si bien es cierto que la sociedad se manifiesta progresivamente más respetuosa con el hecho de la discapacidad, todavía hoy, e incluso en la propia universidad, escuchamos términos que denotan cierto desconocimiento de esta realidad y que, en la mayoría de los casos, se muestran sobreprotectores e, incluso, ofensivos para las personas con SD a las que se refieren (MOLINA; ILLÁN, 2011; STAINBACK; STAINBACK; EAST; SAPON-SHEVIN, 1994). Las actitudes que siempre han despertado las personas con SD, han estado marcadas, principalmente, por la idea de irracionalidad y rechazo. Consecuentemente, estas personas han sido percibidas como un estorbo para la sociedad. Esta consideración justifica las continuas oscilaciones de una actitud colectiva que gravita entre la tolerancia y la reprobación, la integración y la segregación (BARON; BYRNE; 1998; INE, 1999). A pesar de que las transformaciones sociales y culturales han ido modificando las representaciones colectivas sobre el SD, las antiguas concepciones siempre han subsistido, en cierta medida, entremezcladas con los nuevos conocimientos científicos y con nuevas formas semánticas de referirnos al mismo hecho. Sabemos que todavía persisten actitudes negativas hacia las personas con SD (GARCÍA; SARABIA, 2001; GLENN; CUNNINGHAM, 2001; GÚTIEZ, 2003), y que dicha actitud tiene un efecto perjudicial en la vida de las personas con discapacidad intelectual. Y lo que resulta más importante, hemos experimentado el efecto de malas actitudes a través de los escasos niveles de interés, apoyo y compromiso social que encontramos, frecuentemente, con relación a este particular (MOLINA; ILLÁN, 2008).

En ese sentido, la idea de incluir a las personas con SD en todos los aspectos de la vida cotidiana, es continuamente desafiada por las actitudes negativas y estereotipos de una sociedad hegemónica (IZUZQUIZA, 2003). Las actitudes y expectativas de la sociedad determinan el grado hasta el cual las personas con SD pueden aprender, desarrollar, amar, vivir o trabajar. Aunque la inclusión, por lo menos como filosofía, se ha vuelto ampliamente aceptada (FARIA; 2005; GILSON; COOKE; MAHONEY, 2005; STONE; MAY, 2002), la aceptación total de las personas con SD bien pareciera no haberse logrado aún. Hacia esta clarificación camina este trabajo, en concreto centrando nuestra atención en la percepción que de ellos tienen los futuros maestros, por tratarse de un cuerpo profesional que, a corto o medio plazo, tendrán que dedicar su ejercicio laboral para con este grupo de población. Teniendo en cuenta que de su percepción dependerá, en gran medida, la calidad de vida futura de estas personas, hemos decidido centrar nuestra atención por abordar este estudio. Para ello, hemos diseñado una escala a través de la cual podamos determinar cuáles son las percepciones sociales que, con respecto al SD, se dan entre los estudiantes universitarios del título de Magisterio (especialidad de Educación Física) de la Universidad de Murcia, por tratarse de futuros profesionales que interactuarán con este 
colectivo, y que han cursado un plan de estudios que contiene una formación universitaria genérica y específica sobre este particular. Partiendo del entendimiento de las barreras actitudinales de la inclusión de estas personas en el seno de un gran número de ámbitos sociales, pretendemos documentar las percepciones de la sociedad hacia las personas con SD. Tras un análisis de la literatura especializada (CERIGNONI; DE SOUZA; HUNGER, 2008; MANOEL; DE CARVALHO, 2011; TORRI; STAIMBACH; FERNANDEZ, 2007), el estudio se centra, precisamente, en la validación de la escala a partir de las percepciones de este colectivo universitario sobre las capacidades de las personas con SD, sus creencias sobre dónde y cómo deben vivir, trabajar, desarrollar su sexualidad y/o formarse, así como sus creencias y expectativas sobre los obstáculos y consecuencias de la inclusión. Se trata de una investigación financiada por el Ministerio de Educación, la Fundación para el SD (FUNDOWN), la Fundación Séneca y la Fundación Esteban Romero.

\section{Marco teórico}

Nuestro trabajo discurre alrededor de dos grandes bloques de estudio. Por un lado el de la percepción y, por otro, el de la discapacidad intelectual (SD). En primer lugar, hablaremos sobre el término percepción y, posteriormente, haremos referencia a la nomenclatura que gira - y ha girado - en torno al concepto de discapacidad. El concepto de percepción social ha sido uno de los temas más importantes para la Psicología Social, hasta el punto de convertirse en su objeto de estudio (JASPAR; HEWSTONE, 1985). Nuestro trabajo se nutre de esta joven rama de la Psicología, cuyo enfoque de estudio es, entre otros, la percepción de las personas. Esta vertiente de la Psicología no hizo su aparición hasta principios del siglo XX. Turner (1999, p. 2), define a la Psicología Social, y a su objeto de estudio, del modo siguiente:

La Psicología es la ciencia de la mente y la Psicología social es la ciencia de los aspectos sociales de la vida mental [...] es el funcionamiento de la mente individual en sociedad.

Pero ¿qué entendemos por percepción social? La percepción social se podría definir como el proceso a través del cual pretendemos conocer y comprender a los demás (BARON; BYRNE 1998). El acto de comprender el comportamiento de los demás (el por qué de sus actos), ha sido uno de los temas más importantes para esta ciencia. Como afırman Jaspar y Hewstone (1985),

sería difícil explicar la acción humana y, sin duda, el comportamiento social sin un conocimiento de los procesos cognitivos que sirven como mediadores entre la realidad física u objetiva y la reacción del individuo. (p. 415)

Han sido numerosos los estudios que se han realizado en base a la percepción social: Teoría de la formación de impresiones de Asch (1946); teorías sobre la atribución (HEIDER, 1958; JONES; DAVIS, 1965; KELLEY, 1967); teorías implícitas de la personalidad como la de Bruner \&t Tagiuri (1954) etc. Pero el término percepción social sufrió un cambio de nomenclatura. Las diversas investigaciones en este campo, hicieron que este término pasara a denominarse cognición social (Turner, 1999, explica cómo ha sido este cambio). En este sentido, Baron y Byrne (1998), hacen también referencia al cambio de nomenclatura que han adoptado las investigaciones sobre la percepción social. Por otra parte, y según Leyens y Codol (1993), la percepción social está considerada como la base de la cognición social, al igual que todas las teorías en las que se ha fundamentado (teoría de Asch, Bruner, Tagiuri y Heider, entre otras). Estos autores, definen el término de cognición social como el proceso mental por el cual el ser humano recibe, selecciona, transforma y organiza la información del exterior para crear conocimiento. Para estos autores, seleccionar la información que recibimos de nuestro entorno 
(ya sea de nuestro sentidos, recuerdos o de conversaciones con otras personas), es uno de los pasos fundamentales para obtener conocimiento. El conocimiento se concibe aquí como la piedra angular para conseguir una buena adaptación al medio. Cuanto más conozcamos al medio, cuanto más lo comprendamos, mejor nos adaptaremos a él. Como hemos visto, son varios los autores que abogan por la utilización del término cognición frente al de percepción. No obstante, existen también autores más recientes que optan por utilizar el término de percepción como sinónimo de cognición (MOYA, 1999). En ese sentido, nos hemos sumado a esta consideración en la medida en que dicho término se refiere a la manera en que las personas comprendemos, concebimos o interpretamos el mundo social que nos rodea. Por ello, elementos como estereotipos, prejuicios, normas, actitudes y representaciones sociales, son algunos de los temas que forman parte de nuestra fundamentación teórica en este campo.

En segundo lugar, también abordamos el concepto discapacidad intelectual. Se trata de un constructo cargado de ideología y nutrido de diferentes terminologías - algunas poco acertadas - para referirse a las personas con discapacidad (TOMLINSON, 1982). Desde 1886 hasta la llegada del Informe Warnock (1978), existe un claro predominio de términos despectivos y groseros para hacer referencia, en general, a las personas con discapacidad. En particular, al hablar de las personas con discapacidad intelectual, se les ha nombrado con términos clínicos como idiota, cretino, imbécil, o subnormal. No sería hasta la llegada del Informe Warnock (1978) cuando se les denominase como personas con necesidades educativas especiales. Este informe pone su énfasis en la carencia educativa, así como en la importancia que la respuesta educativa debe proporcionar al entorno social de las personas con discapacidad. Con este nuevo informe, no sólo se cambia la nomenclatura, sino que también cambiamos el enfoque. Se pasa, así, de un enfoque médico y clínico, donde la etiquetación era una medida necesaria para acabar con la, supuesta, enfermedad, a un nuevo enfoque donde las personas con discapacidad eran consideradas como sujetos que necesitan una medidas de atención, las cuales deben de ser cubiertas por el medio social en el que viven. De esta manera, el centro de atención ya no es el déficit, sino que las miradas se centran en las capacidades de la persona. A pesar de este cambio revolucionario que supuso el Informe Warnock (GUTIÉZ, 2003), el Centro Nacional de Recursos de Educación Especial afirmó que todavía se seguía haciendo más hincapié en el déficit que en las capacidades. Izuzquiza (2003) recoge el pensamiento de Scheerenberger (1984) cuando se refiere al uso de los diversos términos que han sido asignados a la discapacidad (idiota, imbécil, retrasado). Esta gran amplitud terminológica, se convierte en piedra angular para que la Organización Mundial de la Salud (OMS) creara la primera Clasificación Internacional de Deficiencias, Discapacidades y Minusvalías (CIDDM, 1980). De esta manera, se elabora una nomenclatura - común e internacional - a la hora de hacer referencia a la discapacidad, cuyo periodo de gestación culminó con el alumbramiento de términos como deficiencia, discapacidad y minusvalia, todos ellos basados en la consecución o proceso de una enfermedad. En ese sentido, el término deficiencia se refiere al estado de pérdida de una estructura del cuerpo o de una función del mismo, provocando lo que se denomina una restricción o discapacidad, en tanto en cuanto una estructura u órgano no desempeña la función fisiológica para la que ha sido concebida. Esta circunstancia es, precisamente, la que genera una limitación en la persona a la hora de desempeñar un rol social, el cual queda entendido - por la sociedad en su conjunto- como algo natural, en función de las características del grupo al que pertenezca dicho sujeto (según su género, su edad, su entorno etc.), diciéndose, entonces, que tiene una minusvalía, a causa, precisamente, de no poder desempeñar dicho papel socialmente legitimado y establecido. Como era de esperar, esta clasificación recibió críticas desde diferentes sectores, hecho que condujo a su revisión, dando lugar a una nueva clasificación (también llamada CIDDM2), denominada en 2001 como Clasificación Internacional del 
Funcionamiento de la Discapacidad y de la Salud (CIF). A diferencia de la anterior, la nueva clasificación centra su atención en la salud y no en la enfermedad. Esta nueva escala trata de poner en positivo su nomenclatura, de ahí que aparezcan nuevos términos (como el de funcionamiento), cuya finalidad es dar cobertura e incorporar todas las funciones y estructuras fisiológicas que permiten la participación social del individuo. Por último, aparece el término discapacidad, como concepto genérico, para recoger las deficiencias en las estructuras y funciones corporales, amén de las limitaciones a la hora de desempeñar un rol social (GARCÍA; SARABIA, 2001). Como hemos visto, el concepto discapacidad es un término reciente. Antes de la década de 1980, el término empleado - en este contexto - era retraso mental. Como cita Verdugo (2002):

La principal razón para sugerir un cambio en la terminología, se deriva del carácter peyorativo del significado de retraso mental que, además, reduce la compresión de las personas con limitaciones intelectuales a una categoría diagnóstica nacida desde perspectivas psicopatológicas. Las discapacidad intelectual debe concebirse hoy desde un enfoque que subraye, en primer lugar, a la persona como a cualquier otro individuo de nuestra sociedad. (p. 12)

En años posteriores a la primera clasificación, surgieron diferentes manuales hablando, literalmente, del retrasado mental, clasificándolo a tenor de su cociente intelectual (C.I.). De entre ellos, queremos destacar tres: el Manual Diagnóstico y Estadístico de los Trastornos Mentales (DSM-IV) - cuya autoría pertenece a la Asociación Americana de Psiquiatría (APA, 1995); el manual de la Asociación Americana de Retaso Mental (AMMR, 1992) y, por último, el Manual de los Trastornos Mentales y del Comportamiento (CIE-10), creado por la Organización Mundial de la Salud (OMS), entre otros. Pero sería, realmente, la labor realizada por la AAMR (en su novena edición de 1992), quien imprimiría un cambio radical en lo que venía siendo el paradigma tradicional de funcionamiento, alejándose de la concepción de retrasado mental como mero aspecto individual, pasando a tener en consideración la interacción entre la persona y el medio que la rodea. En su nueva versión (2002), la AAMR intenta evaluar más allá de los parámetros recogidos en el cociente intelectual, dando cabida tanto a las habilidades sociales como prácticas que permiten garantizar una integración social satisfactoria. En este sentido, podemos afirmar que este es uno de los planteamientos, de partida, que nutren nuestras inquietudes a la hora de realizar un estudio sobre integración social, de la naturaleza del que estamos realizando. Todo este clima, supuso un gran avance que adicionar a la labor emprendida, anteriormente, por la Asociación Internacional para el Estudio Científico de la Discapacidad Intelectual (IASSID), que en 1964 ya proponía el término discapacidad intelectual frente a retrasado mental. En nuestro país, la Federación Española de Asociaciones pro Subnormales (FEAPS), propuso también la utilización del término de discapacidad, cambiando incluso su propia designación por la de Confederación Española de Organizaciones a favor de Personas con Discapacidad Intelectual. No podemos concluir este epígrafe, sin indicar que el término discapacidad intelectual ha quedado incluido, desde enero de 2007, en la misma nomenclatura de la Asociación Americana de Retraso Mental, pasando a denominarse American Association on Intellectual and Developmental Disabilities (Asociación Americana sobre Discapacidades Intelectuales y del Desarrollo).

\section{Material y método}

El presente trabajo tiene por objetivo principal la construcción y validación de una escala de fácil aplicación (denominada EPSD-1), que permita conocer y analizar la percepción social que mantienen los estudiantes universitarios sobre las personas con SD. Desde este planteamiento, pretendemos, de forma general, conocer las opiniones, creencias y actitudes de los estudiantes del título de Educación Física sobre las personas 
con SD, precisamente por tratarse de un colectivo de profesionales que, potencialmente, habrá de trabajar, en un futuro, con personas con SD, tanto en el ámbito de la educación formal como no formal (siguiendo la tendencia de mercado actual). Precisamente porque consideramos que se trata de un numeroso colectivo y porque su influencia social está resultando esencial para proyectar una imagen (positiva o negativa) del SD, decidimos concentrar en ellos nuestras energías (al menos en esta fase inicial). Como objetivo subsidiario estaría conocer y analizar la percepción existente sobre el grado de integración social de las personas con SD. Nuestro propósito futuro se centrará en la realización de otro estudio a partir del instrumento validado aplicado a diferentes contextos.

Este estudio ha sido realizado siguiendo tres grandes etapas: construcción de los ítems de la escala, análisis de jueces y validación del instrumento de recogida de información. A continuación, presentaremos los procedimientos y materiales utilizados en cada una de las diferentes fases de investigación, así como los escenarios y personas involucradas en cada una de ellas.

Los items de la EPSD-1 se elaboraron partiendo de una doble vía. En primer lugar, mediante un exhaustivo análisis documental sobre los grandes ámbitos emergentes relacionados con la integración y calidad de vida de las personas con SD (ARIAS, 2004; JIMÉNEZ, 2001; MOLINA; ILLÁN, 2008). En segundo lugar, a partir de un proceso de consultoría con expertos, de reconocido prestigio, dedicados al ámbito de la discapacidad intelectual. De este modo, diseñamos una escala con 45 items organizados alrededor de los siete ámbitos más influyentes recogidos en la literatura (GLENN; CUNNINGHAM, 2001; MOLINA; ILLÁN, 2011; SPIJKER; PÉREZ, 2010): salud, ocio y tiempo libre, derechos y deberes, sexualidad, empleo, integración social y educación. Obtuvimos así una escala completamente original, construida a partir de la revisión de diversas investigaciones sobre la percepción social de la discapacidad, física, sensorial y metabólica (BERNARDO; MATOS, 2003; CORREDEIRA et al., 2007; CROCKER; EKLUND; KOWALSKY, 2000), conjuntamente con el aporte de los expertos en la materia. Esta escala - y todos los itemes que la conforman fueron sometidos a la opinión de trece expertos en discapacidad intelectual, con reconocido prestigio en el ámbito nacional e internacional, procedentes de centros de investigación superior, centros sanitarios, asociaciones y Ministerio, entre otros. Una vez diseñado el instrumento, sometimos su contenido al juicio de un grupo de expertos, con el fin de evaluar la pertinencia de las cuestiones elaboradas, el grado de acierto en las dimensiones definidas, así como también la comprensión y adecuación semántica de cada item. En total, el grupo de jueces estaba compuesto por 13 profesionales en activo y de reconocido prestigio en los ámbitos de metodología de la investigación, percepción social y discapacidad. Como criterio para conservar un ítem, utilizamos un grado de concordancia de, al menos, el 75\% de los jueces. Como resultado de este proceso, de los 40 ítems iniciales se descartaron 9, conservándose únicamente 31, precisamente por ser estos los que mejor describían la percepción social del SD. Del mismo modo, los treinta y un indicadores finales sufrieron también un proceso de depuración y alteración de su estructura gramatical original, a tenor de las diferentes propuestas de los jueces. Los 31 ítems se organizaron, sistemáticamente, en un cuestionario estructurado en los siete ámbitos citados anteriormente (salud, ocio y tiempo libre, derechos y deberes, sexualidad, empleo, integración social y educación), con un espacio reservado para la codificación de las variables independientes. Las respuestas se organizaban utilizando una escala de tipo Likert de cinco opciones (1- Totalmente de acuerdo; 2- De acuerdo; 3- Indiferente; 4- En deascuerdo; 5- Totalmente en desacuerdo).

A continuación, vamos a diferenciar dos aspectos esenciales en el proceso de validación: la muestra y el procedimiento de aplicación. Una vez realizado el procedimiento para constatar la validez semántica del instrumento (a través del análisis de jueces), realizamos un estudio piloto con el fin de realizar la validación de contenido. Para ello, utilizamos una muestra de 1.796 sujetos, todos ellos estudiantes de todos los cursos 
de los títulos de Educación Física pertenecientes a las dos universidades que imparten dicho título en la Región de Murcia (Universidad de Murcia - UMU y Universidad Católica San Antonio de Murcia - UCAM). Una vez descartados todos los cuestionarios que arrojaron errores, la muestra final quedó compuesta por 1.312 sujetos, entre los cuales predomina, ligeramente, la presencia de los hombres (56\%) frente a las mujeres (44\%), con edades comprendidas entre los 18 y los 35 años. Como podemos ver en el siguiente cuadro (tabla 1), llama la atención que del total de la muestra, casi el 4\% ha declarado tener discapacidad (física, en su mayoría), al tiempo que el $44 \%$ afirma tener algún familiar con discapacidad.

Tabla 1- Estadísticos descriptivos de la muestra

\begin{tabular}{l|c:c}
\hline Sexo & Frecuencia & Porcentaje \\
\hline Masculino & 574 & 43,8 \\
\hline Femenino & 738 & 56,3 \\
\hline Total & 1312 & 100,0 \\
\hline Discapacidad & Frecuencia & Porcentaje \\
\hline SI & 44 & 3,4 \\
\hline NO & 1268 & 96,6 \\
\hline Total & 1312 & 100,0 \\
\hline Tipo de discapacidad & Frecuencia & Porcentaje \\
\hline Intelectual & 3 & 6,8 \\
\hline Física & 34 & 77,3 \\
\hline Sensorial & 3 & 6,8 \\
\hline Física e intelectual & 2 & 4,5 \\
\hline Física y sensorial & 1 & 2,3 \\
\hline Metabólica & 1 & 2,3 \\
\hline Total & 44 & 100,0 \\
\hline Familiar con discapacidad & Frecuencia & Porcentaje \\
\hline Sl & 581 & 44,3 \\
\hline N0 & 731 & 55,7 \\
\hline Total & 1312 & 100,0 \\
\hline
\end{tabular}

Un dato a destacar es que la muestra representa el 94\% de la población total, hecho que concede al estudio un nivel de confianza elevado (superior al 97\% - Programa STATS 2.0), con un error máximo aceptable del 1\% (HERNÁNDEZ; FERNÁNDEZ-COLLADO; BAPTISTA, 1998). Para calcular el nivel de confiabilidad del instrumento, hemos utilizado el método de Split-Half, junto con el método de Kider-Richardson. Con relación al primero de ellos, calificamos por separado lo items pares e impares, correlacionando las series de puntuaciones resultantes con el coeficiente $r$ corregido de Pearson, a través de la fórmula de profecía de Spearman-Brown. Con relación al método de equivalencia racional, para calcular la confiabilidad interna del instrumento, aplicamos el coeficiente $\alpha$ de Cronbach para cada uno de los factores. Siguiendo a Malhotra (2001), hemos descartado todos los ítems cuyos coeficientes $\alpha$ extraídos no alcanzaban los valores de referencia para una confiabilidad satisfactoria. En nuestro caso, hemos obtenido un valor del coeficiente $\alpha$ de 0,817 para toda la escala, hecho que, según Malhotra (2001), le confiere una elevada fiabilidad. Otro de los aspectos importantes para la configuración definitiva de esta escala, fueron los criterios de aplicabilidad y eficiencia (HASTAD; LACY, 1998), que también hemos aplicado, pues dada la elevada muestra con la que hemos trabajado, nos interesaba que el instrumento fuese de fácil aplicación y con un consumo reducido de tiempo por parte del sujeto.

Para la aplicación del instrumento, solicitamos el permiso oportuno a las autoridades académicas responsables. Concedido este, el procedimiento consistió en distribuir los cuestionarios a los profesores responsables de la docencia en los grupos en los que se organizaba la muestra, aplicándose directamente en el aula y de forma voluntaria para el alumnado. Por otra parte, y con el objetivo de asegurar un elevado nivel de confianza en los resultados, realizamos una versión digital del cuestionario, con el propósito de que todos aquellos estudiantes que no hubiesen podido asistir el día de la prueba presencial, tuviesen la oportunidad de realizarlo y hacérnoslo llegar. Para la elaboración de este instrumento digital contamos con el asesoramiento de los expertos del Servicio de Apoyo a la Investigación de la Universidad de Murcia. El resultado fue un cuestionario que se permitía rellenar de forma digital (y en tiempo real) o bien imprimir y contestar de forma analógica. En el primer caso, se generaba un informe automático de respuesta con los datos contenidos. En el segundo caso, se trataba de un documento impreso de lectura óptica, cuyo vaciado de la información lo realizaría posteriormente un escáner. 


\section{Resultados}

Las respuestas contenidas en los cuestionarios las organizamos formando un banco de datos, el cual - debidamente codificado constituye una plantilla de trabajo en el programa SPSS (Statistical Package of Social Sciences), con el que hemos analizado la información. Para el análisis de todos los datos, también hemos contado con la colaboración de los expertos del servicio de cálculo científico y apoyo estadístico de la Universidad de Murcia. Para desarrollar este estudio, hemos llevado a cabo un análisis factorial exploratorio de la escala EPSD-1. Dado que la formulación de determinados ítems se realiza tanto en positivo como también en negativo, para el análisis de los datos todas las puntuaciones de los itemes que indican rechazo social se han invertido con objeto de poder conjugarlos, adecuadamente, con los valores de aquellos ítems que indican aceptación social (por ejemplo, se han invertido los ítems $8,21,25,26)$. A través de este análisis factorial, nuestro objetivo ha sido extraer los factores comunes (o variables latentes) que explican la percepción social del SD presente en la EPSD-1, con objeto de validar esta escala para su aplicación a diferentes contextos. Siguiendo a Pérez (2005), hemos utilizado el método de extracción de componentes principales sobre la matriz de covarianzas, con objeto de formar combinaciones lineales no correlacionadas de las variables observadas. Hemos rotado la solución factorial mediante rotación varimax, minimizando así el número de variables que tienen saturaciones altas en cada factor, y simplificando la interpretación de los mismos. La solución factorial la hemos realizado sin determinar el número máximo de factores, por lo que el criterio fue aquellos cuyo eigenvalue fuese superior a 1 . Hemos obtenido un total de diez factores, como se puede apreciar en el gráfico de sedimentación de la imagen (Figura 1).

Figura 1- Gráfico de sedimentación

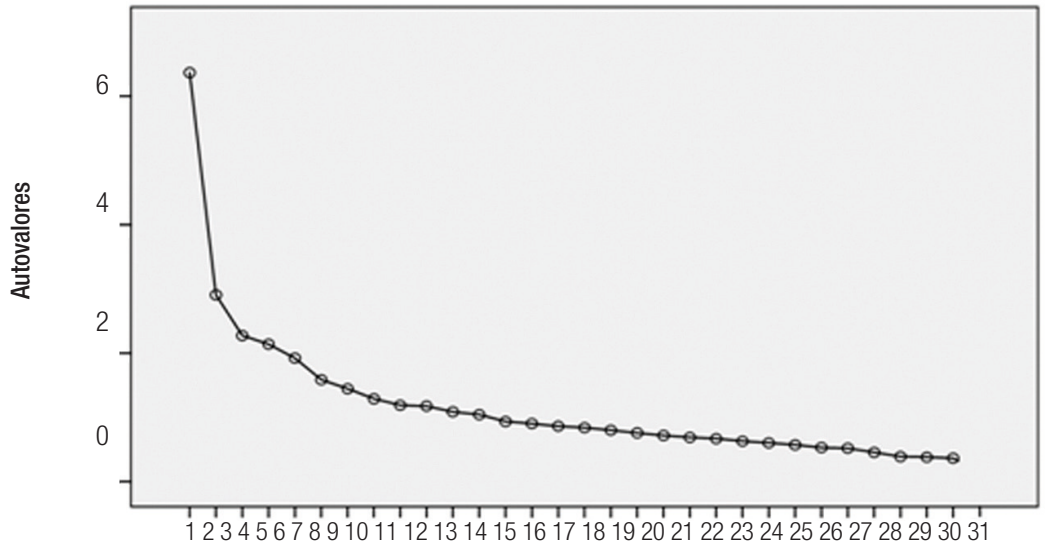

Número de componente

La prueba de esfericidad de Barlett indica que la matriz de correlaciones cumple con el requisito de que los items estén intercorrelacionados entre sí, siendo Chi-cuadrado $(465)=9861.99 ; p=0,000$. En el cuadro siguiente (tabla 2), recogemos los estadísticos descriptivos de los factores extraídos:

Tabla 2 - Estadísticos descriptivos de los factores

\begin{tabular}{c|c:c:c}
\hline & $\mathbf{N}$ & Media & Desviación típica \\
\hline F1 & 1312 & 40,745 & 5,388 \\
\hline F2 & 1312 & 16,696 & 2,192 \\
\hline F3 & 1312 & 6,090 & 2,182 \\
\hline F4 & 1312 & 7,073 & 1,812 \\
\hline F5 & 1312 & 6,264 & 1,329 \\
\hline F6 & 1312 & 4,786 & 1,893 \\
\hline F7 & 1312 & 5,666 & 1,844 \\
\hline F8 & 1312 & 6,323 & 1,819 \\
\hline F9 & 1312 & 6,136 & 1,478 \\
\hline F10 & 1312 & 5,809 & 1,742 \\
\hline
\end{tabular}


Por otra parte, la medida de adecuación muestral de Kaiser-Meiyer-Olkin (KMO), indica que las características de la muestra son adecuadas para realizar un análisis factorial, adoptando KMO un valor de 0,846.

Tabla 3 - KMO y prueba de Barlett

\begin{tabular}{llll}
\hline \multicolumn{2}{l}{ Medida de adecuación muestral (KMO) } & $\vdots$ & 0,846 \\
\hline \multirow{3}{*}{$\begin{array}{l}\text { Prueba de esfericidad } \\
\text { de Bartlett }\end{array}$} & Chi-cuadrado aproximado & Gl. & 9861,99 \\
& $\vdots$ & $\vdots$ & 465 \\
\hline
\end{tabular}

a. Basado en correlaciones

Esto también se ve confirmado por la diagonal de la matriz de correlaciones antiimagen, con valores próximos a la unidad. En total, los diez factores cuyos eigenvalues son superiores a 1, explican en la matriz reescalada de varianzas el $84.11 \%$ de la varianza total (tabla 4).

Tabla 4- Varianza total explicada tras rotación de los factores seleccionados: matriz reescalada

\begin{tabular}{|c|c|c|c|c|}
\hline & \multirow[b]{2}{*}{ Componente } & \multicolumn{3}{|c|}{$\begin{array}{c}\text { Suma de las saturaciones al } \\
\text { cuadrado de la rotación }\end{array}$} \\
\hline & & Total & $\begin{array}{c}\% \text { de la } \\
\text { varianza }\end{array}$ & $\begin{array}{c}\% \\
\text { acumulado }\end{array}$ \\
\hline \multirow{10}{*}{ Reescalada } & 1 & 4,28 & 16,33 & 16,33 \\
\hline & 2 & 2,03 & 9,06 & 25,39 \\
\hline & 3 & 1,98 & 8,91 & 34,3 \\
\hline & 4 & 1,61 & 7,71 & 42,01 \\
\hline & 5 & 1,59 & 7,64 & 49,65 \\
\hline & 6 & 1,59 & 7,63 & 57,28 \\
\hline & 7 & 1,41 & 7,06 & 64,34 \\
\hline & 8 & 1,27 & 6,62 & 70,96 \\
\hline & 9 & 1,27 & 6,59 & 77,55 \\
\hline & 10 & 1,26 & 6,56 & 84,11 \\
\hline
\end{tabular}

A continuación, presentamos los componentes extraídos. El primer factor (1), que hemos denominado de exclusión social o marginación de las personas con SD, está formado por diez ítems que indican el rechazo y la incomodidad a compartir actividades sociales y de ocio con personas con SD (explica el 16.33\% de la varianza total). A continuación, ofrecemos la matriz de componentes rotados, que recogen los ítems pertenecientes a cada uno de los diferentes factores (tabla 5):
Tabla 5. Matriz de componentes rotados (factores)

\begin{tabular}{|c|c|}
\hline Factor 1: Exclusión social o marginación de las personas con SD & $\%$ \\
\hline Me desagrada compartir lugares de ocio con personas con SD, & 0,69 \\
\hline Me incomoda que una persona con SD se me acerque & 0,67 \\
\hline Si una persona con SD se acerca, le doy atención/conversación & $-0,37$ \\
\hline No participaría en un viaje organizado con personas con SD. & 0,69 \\
\hline $\begin{array}{l}\text { Es necesario prohibir que las personas con SD tengan relaciones } \\
\text { sexuales. }\end{array}$ & 0,47 \\
\hline $\begin{array}{l}\text { Las personas con SD pueden tener relaciones sexuales sólo con otras } \\
\text { personas con SD. }\end{array}$ & 0,50 \\
\hline Me incomodaría la presencia en mi trabajo de personas con SD. & 0,63 \\
\hline Sentiría vergüenza paseando por la calle junto a personas con SD. & 0,65 \\
\hline Me molestaría que mi hijo tuviese compañeros de clase con SD. & 0,60 \\
\hline Si fuese maestro, preferiría no tener alumnos con SD. & 0,49 \\
\hline \multicolumn{2}{|l|}{$\begin{array}{l}\text { Factor 2: Rechazo de la autonomía e independencia de las personas } \\
\text { con SD }\end{array}$} \\
\hline Las personas con SD no saben disfrutar el ocio como los demás & 0,453 \\
\hline Las personas con SD no están preparadas vivir en pareja & 0,491 \\
\hline $\begin{array}{l}\text { Las personas con SD tienen derecho a decidir dónde, cómo, con quién } \\
\text { vivir }\end{array}$ & $-0,486$ \\
\hline No debería permitirse que las personas con SD tengan o adopten niños: & 0,610 \\
\hline $\begin{array}{l}\text { Las personas con SD están preparadas para educar/cuidar niños cor } \\
\text { otra persona }\end{array}$ & $-0,526$ \\
\hline \multicolumn{2}{|l|}{$\begin{array}{l}\text { Factor 3: Rechazo a mantener relaciones afectivo/sexuales con } \\
\text { personas con SD }\end{array}$} \\
\hline Nunca sentiría deseo sexual por una persona con SD & 0,917 \\
\hline Nunca me enamoraría de una persona con SD & 0,914 \\
\hline \multicolumn{2}{|l|}{ Factor 4: Aceptación social en el ámbito educativo. } \\
\hline $\begin{array}{l}\text { Los niños con SD deben estudiar en mismas escuelas que resto de } \\
\text { niños. }\end{array}$ & 0,839 \\
\hline $\begin{array}{l}\text { Si mi hijo estudiase en una escuela con niños con SD, estos } \\
\text { enriquecerían su vida. }\end{array}$ & 0,580 \\
\hline \multicolumn{2}{|l|}{ Factor 5: Aceptación a tener descendencia con SD } \\
\hline Si mi hijo tuviese SD abortaría. & 0,865 \\
\hline Rechazo a que mis padres/pareja abortasen un bebé con SD & $-0,832$ \\
\hline \multicolumn{2}{|l|}{ Factor 6: Control externo de la sexualidad. } \\
\hline $\begin{array}{l}\text { Control de sexualidad de las personas con SD mediante familiares y } \\
\text { expertos. }\end{array}$ & 0,696 \\
\hline $\begin{array}{l}\text { Las personas con SD pueden tener relaciones sexuales consentidas } \\
\text { por padres y controladas expertos. }\end{array}$ & 0,858 \\
\hline Factor 7: Rechazo de la integración de las personas con SD & \\
\hline $\begin{array}{l}\text { La vida independiente de las personas con SD no está bien vista por } \\
\text { la sociedad. }\end{array}$ & 0,783 \\
\hline Socialmente, incomoda la presencia de personas con SD. & 0,732 \\
\hline \multicolumn{2}{|l|}{ Factor 8: Control anticonceptivo de las personas con SD. } \\
\hline $\begin{array}{l}\text { Anticonceptivos irreversibles son lo más adecuado para que tengan } \\
\text { sexo las personas con SD. }\end{array}$ & 0,648 \\
\hline Anticonceptivos eficaces deben ser usados por las personas con SD. & 0,834 \\
\hline \multicolumn{2}{|l|}{ Factor 9: Proteccionismo hacia las personas con SD. } \\
\hline $\begin{array}{l}\text { El empleo protegido es la única salida profesional para las personas } \\
\text { con SD. }\end{array}$ & 0,460 \\
\hline Si tuviese hijo con SD no condicionaría mi vida. & $-0,742$ \\
\hline \multicolumn{2}{|l|}{$\begin{array}{l}\text { Factor 10: Escasa formación docente y actuación paternalista ante la } \\
\text { integración de personas con SD. }\end{array}$} \\
\hline Los profesores no están preparados para atender a niños con SD. & 0,837 \\
\hline $\begin{array}{l}\text { El maestro siente pena por niños con SD y por ello les facilitan todo y } \\
\text { aprueban }\end{array}$ & 0,404 \\
\hline
\end{tabular}


A modo de resumen, tal y como podemos apreciar, el segundo factor está compuesto por cinco ítems que explican el 9.06\% de la varianza total; puede ser interpretado como rechazo de la autonomía e independencia de las personas con SD, ya que el contenido de los ítems está referido a la capacidad de estas personas para llevar una vida autónoma y de tener descendencia. El tercer factor está formado por dos ítems que explican el 8.91\% de la varianza total, y se puede interpretar como rechazo a mantener relaciones afectivo/ sexuales con personas con SD. El cuarto factor estaría compuesto por dos ítems que explican el $7.71 \%$ de la varianza total y se interpreta como aceptación social en el ámbito educativo. En el ecuador de esta tabla, el quinto factor estaría compuesto por dos ítems que explican el $7.64 \%$ de la varianza total y se interpreta como aceptación a tener descendencia con SD. El sexto factor explica el $7.63 \%$ de la varianza total y se interpreta como control externo de la sexualidad. El séptimo factor se interpreta como rechazo de la integración de las personas con SD; el octavo hace referencia al proteccionismo hacia las personas con SD. El noveno factor está formado por dos items y se interpreta como control anticonceptivo de las personas con SD. Por último, el décimo factor está formado por dos ítems que explican el $6.56 \%$ de la varianza total y se interpreta como escasa formación docente y actuación paternalista ante la integración de personas con SD. Tras realizar el ajuste de los factores, obtenemos una nueva matriz de residuales de las correlaciones reproducidas, la cual nos indica que el porcentaje de la varianza total de las variables observadas explicada en los diez factores es alto $(84,11 \%)$. Además, podemos concluir que la escala EPSD-1 tiene bondad de ajuste, ya que sólo hay un 3\% de residuales con valores absolutos superiores a 0.05. Por último, el coeficiente de consistencia interna (alfa de Cronbach), que mide la fiabilidad y la coherencia interna entre ítems, ha arrojado un valor de 0,871 para toda la escala, mostrando así una elevada calidad. Por lo tanto, la EPSD-1 presenta una estructura factorial adecuada a la hora de evaluar cómo los estudiantes del título de Educación Física perciben a las personas con SD, incluyendo las grandes dimensiones consideradas por diferentes autores (véase ABIATTI, 2004; GUERRINI, 2006; RODRÍGUEZ, 2003; ZUZA, 2000).

\section{Discusión}

Tal y como hemos mostrado, la EPSD1 encierra diez grandes factores alrededor de los cuales se organiza la percepción social que tienen los estudiantes de Magisterio con relación a las personas con SD. El primer factor explicativo en la EPSD-1, recoge determinados aspectos que expresan el rechazo y la incomodidad a compartir actividades sociales y de ocio con personas con SD, explicando el porcentaje acumulado mayor de la varianza total. Los items que constituyen este factor, expresan en qué medida las personas sienten rechazo ante el hecho de compartir lugares de estudio, de trabajo y de ocio con personas con $\mathrm{SD}$, o incluso la incomodidad surgida ante el acercamiento repentino de una persona con SD. Además, también se incluyen ítems referidos a la negativa a dar conversación a estas personas, o a participar en un viaje organizado sabiendo, previamente, que pudieran ser compañeros de viaje. También forman parte de este factor varios ítems referidos a la necesidad de prohibir las relaciones sexuales de las personas con SD o limitarlas a prácticas, exclusivamente cerradas, entre otras también con dicho síndrome. Por último, se le suman dos ítems muy importantes para la percepción de la función docente, referidos al rechazo (como maestro) a tener alumnos con SD en el aula e, incluso, al hecho de sentirse molesto en el caso de tener hijos compartiendo clase con niños con este síndrome. En general, este factor agrupa toda una serie de indicadores que tendrán como resultado una buena radiografía sobre la percepción social hacia las personas con SD, en aspectos 
tan importantes como el ocio, la educación, el empleo y la sexualidad (CORREDEIRA, 2001; GARCÍA, 1995). El segundo factor, agrupa un conjunto de indicadores referidos a tres grandes ámbitos: derechos, vida en pareja y ocio. Con relación al primer ámbito, se incluyen ítems que recogen la percepción social sobre el derecho que tienen las personas con SD a decidir dónde y con quién desean vivir, así como el derecho a adoptar hijos. En el segundo ámbito, los ítems incluidos hacen referencia a la preparación que las personas con SD tienen para vivir en pareja (incluyendo el cuidado de niños). Por último, este factor también incorpora un ítem referido a si las personas con SD saben (o no) disfrutar de un tiempo de ocio. Qué duda cabe, todo ello supone una concepción social concreta sobre la vida autónoma e independiente (ELIAS; VERMEER; T'HART, 2005; FARR, 2003; GARCÍA; MADARIAGA, 2003). Existen otros dos ítems referidos al hecho de sentir (o no) atracción sexual hacia una persona con $\mathrm{SD}$, o a la posibilidad de enamorarse de ella, convirtiéndose en un factor explicativo de buena parte de la varianza total del estudio. Se trata de una información valiosa, desde el momento en que condiciona nuestra percepción afectiva hacia este colectivo (TALLIS, 2005). Para abordar el ámbito educativo, existen otros dos ítems que recogen la percepción sobre el hecho de que alumnos con y sin síndrome de Down puedan estudiar juntos en la misma escuela, considerando la cuestión del derecho a hacerlo y también de la existencia (o no) de un enriquecimiento mutuo. El quinto de los factores pone al descubierto un tema bastante polémico: el aborto de un niño/a con discapacidad intelectual (CURDOVÁ; VERMEER; VÁLKOVÁ, 2001; GABRIELA, 2004). Se trata, además, de posicionarse a favor o en contra de este tema sabiendo, previamente, que de seguir un embarazo ordinario estaríamos hablando del nacimiento de un bebé con SD. En función de hacia dónde se orienten las respuestas, y conjugadas con otros factores, convendremos que se trata, sin duda, de un tema bastante relevante dentro de esta escala. Directamente relacionado con el anterior, un sexto factor hace referencia a la necesidad de controlar las relaciones sexuales de las personas con SD a través de la intervención familiar y de expertos. Se trata de un tema igualmente polémico. También existe un apartado dedicado al rechazo de la integración de las personas con $\mathrm{SD}$, recogido en el factor 7 , a través del cual se recoge la percepción social negativa hacia la vida independiente de estas personas e, incluso, a la incomodidad que genera su presencia en un entorno social. Relacionado también con el sexto factor, el octavo recoge la recomendación sobre el uso de métodos anticonceptivos eficaces y/o irreversibles, lo que se interpreta como un control anticonceptivo de las personas con SD. Llegando casi al final de nuestro recorrido, el noveno factor está formado por dos ítems que interpretamos como el proteccionismo hacia las personas con SD, a través de los cuales se destaca el empleo protegido como única salida viable para estas personas, junto con un sentimiento: tener un hijo con SD no supone tener que ver tu vida condicionada a tal hecho. El último factor pone de manifiesto la escasa formación del profesorado para trabajar con alumnos con $\mathrm{SD}$, así como la presencia de una actuación paternalista hacia ellos (en la escuela), según la cual todo se les facilita y adapta (en exceso) para que vayan promocionando de curso.

\section{Conclusiones}

A modo de reflexión final, podemos decir que cuando hablamos del proceso de construcción de la percepción, hacemos referencia a un importante componente social, desde el punto de vista de que no miramos desde la nada (desde el vacío), sino que lo hacemos a través de una enraizada herencia cultural, según la cual ordenamos nuestro pensamiento hacia el qué y cómo debemos percibir y, al mismo tiempo, hacia el qué y cómo debemos omitir en dicho proceso (EBBECK; WEISS, 1998; GILSON; COOKE; MAHONEY, 2005). 
Este comportamiento cultural es aprendido socialmente, motivo por el cual está sujeto a modificaciones y cambios. El sistema educativo, y esencialmente la universidad, comparten la responsabilidad en el proceso de construcción y atribución de significados que los futuros maestros realizan ante los diferentes escenarios que perciben. En concreto, orientar la percepción de las diferentes capacidades (y no sólo las mal llamadas discapacidades), es una tarea esencial de la universidad que debe completarse, precisamente, con la propia aportación de la opinión y experiencia de las personas (como en este caso) con SD. Desde el punto de nuestro estudio, las propiedades psicométricas de la EPSD-1 son altamente satisfactorias. Por este motivo, nos hemos planteado realizar nuevos estudios longitudinales y transversales a partir del uso de la EPSD-1 aplicada a diferentes contextos, con objeto de conocer, reflexionar y cuestionar, en gran medida, sobre la práctica docente universitaria, los contenidos, los planes de formación, las estrategias metodológicas adoptadas etc., en tanto en cuando contribuyan a formar a futuros maestros desde una práctica profesional que encierre y taxonomice a los alumnos en categorías diagnósticas que, a su vez, los oprimen, ocultan y homogeneizan. En este sentido, y en función de los futuros resultados, podríamos llegar a conocer la - posible influencia de la formación universitaria para la ulterior representación social del SD, al tiempo que ofrecer una serie de pautas o formas de actuación para los profesionales de la Educación, en general, y la Educación Física en particular, de cara a ir sensibilizando a la sociedad hacia un cambio positivo para la mejora de la imagen social y, por consiguiente, calidad de vida de las personas con SD. A la luz de los resultados obtenidos, consideramos que el instrumento puede resultar de utilidad en el ámbito de las Ciencias Sociales, mostrando una relación latente con respecto a la formación inicial que, desde la universidad, se ofrece a los alumnos de Educación Física. Nuestros posteriores análisis estarán orientados a aplicar este instrumento con objeto de analizar cuál es la percepción actual de los estudiantes y, al mismo tiempo, cuál ha sido la tendencia y evolución al respecto en los últimos años y su posible relación con el proceso de formación universitaria que han seguido.

\section{Referencias}

ABIATTI, Cristina. Es de los ligeros la carrera. Buenos Aires: Altamira, 2004.

AMERICAN ASSOCIATION ON MENTAL RETARDATION. Mental retardation: Definition, classification, and systems of supports (9th ed.). Washington, D. C.: American Association on Mental Retardation, 1992.

ARIAS, José Miguel. Introducción a la investigación en ciencias sociales. In: HIDALGO, Alberto; MEDINA, Raul. (Eds.). Cooperación al desarrollo y bienestar social. Oviedo: Eikasia Ediciones, 2004. p. 87-115.

BARON, Robert; BYRNE, Donn. Psicología Social. Madrid: Prentice Hall, 1998.

BERNARDO, Rui Paulo; MATOS, Margarida Gaspar. Adaptação Portuguesa do Physical Self- Perception Profile for Children and Youth e do Perceived Importance Profile for Children and Youth. Análise Psicológica, Lisboa, v. 2, n. XXI, p. 127-144, 2003.

CÁCERES, Celsa. Sobre el concepto de discapacidad. Una revisión de las propuestas de la OMS. Revista Electrónica de Audiología, Santa Cruz de Tenerife, v. 2, n. 3, p. 74-77, 2004. 
CERIGNONI, Larissa; DE SOUZA, Samuel; HUNGER, Dagmar. 0 processo de constituição histórica das diretrizes curriculares na formação de professores de Educação Física. Educação e Pesquisa, São Paulo, v. 34, n. 2, p. 343-360, 2008.

CORREDEIRA, Rui. Competência percebida e aceitação social em crianças com paralisia cerebral: tradução e adaptação da Pictorial Scale of Perceived Competence and Social Acceptance in Children with Cerebral Palsy para a realidade portuguesa. Tese (Mestrado). Programa de Actividade Física Adaptada, Faculdade de Desporto, Universidade do Porto, Porto, 2001.

CORREDEIRA, Rui; CORTE-REAL, Nuno Jose; DIAS, C.; SILVA, María Adilia; FONSECA, António Manuel. Como avaliar a percepção de competência e aceitação social de crianças com paralisia cerebral? Estudo inicial para a determinação das propriedades psicométricas da versão portuguesa da Dutch Pictorial Scale of Perceived Competence and Social Acceptance in Children with Cerebral Palsy. Revista Brasileira de Educação Especial, São Paulo, v. 13, n. 3, p. 325-344, 2007.

CROCKER, Peter; EKLUND, Robert; KOWALSKY, Kent. Children's physical activity and physical self-perceptions. Journal of Sport Sciences, Glasgow, v. 18, p. 383-394, 2000.

CURDOVÁ, Julie; VERMEER, Adri; VÁLKOVÁ, Hana. Measuring perceived and social acceptance in young children with cerebral palsy: the construction of a Czech Pictorial Scale. Gymnica, v. 1, n. 1, p. 27-36, 2001.

EBBECK, Vicky; WEISS, Maureen. Determinants of children's self-esteem: Influence of perceived competence and affect. Pediatric Exercise Science, Champaign, v. 10, p. 285-298, 1998.

ELIAS, Cristel; VERMEER, Adri.; T' HART, Harm. Measurement of perceived competence in Dutch children with mild intellectual disabilities. Journal of Intellectual Disability Research, Cambridge, v. 49, n, 4, p. 288-295, 2005.

FARIA, Luisa Maria. Desenvolvimento do auto-conceito físico nas crianças e nos adolescentes. Análise Psicológica, Lisboa, v. 23, n. 4, p. 361-371, 2005.

FARR, Robert. De las representaciones colectivas a las representaciones sociales: ida y vuelta. In: CASTORINA, José Antonio. Las representaciones sociales: problemas teóricos y desafíos educativos. Barcelona, Paidós, 2003.

GARCÍA, Carlos; SARABIA, Alicia. Clasificaciones de la OMS sobre discapacidad. 2001. Disponible en: <http://usuarios. discapnet.es/disweb2000/art/ClasificacionesOMSDiscapacidad.pdf>. Acceso en: 20 sept. 2011.

GARCÍA, José Luis. Sexualidad y minusvalías psíquicas: la necesidad de saber. La Cristalera. Madrid, v. 5, p. 44-48, 1995.

GARCÍA, Manuel; MADARIAGA, Aurora. Ocio para todos: reflexiones y experiencias. Deusto: Universidad de Deusto, 2003.

GILSON, Nicholas; Cooke, Carlton; Mahoney, Craig. Adolescent physical selfperceptions, sport/exercise and lifestyle physical activity. Health Education, São Paulo, v. 105, n. 6, p. 437-450, 2005.

GLENN, Sheila; CUNNINGHAM, Cliff. Evaluation of self by young people with Down syndrome. International Journal of Disability, Development and Education, Queensland, v. 48, p. 163-177, 2001.

GRANIZO, Laura; NAYLOR, Paul; DEL BARRIO, Cristina. Análisis de las relaciones sociales de los alumnos con síndrome de Asperger en escuelas integradas de secundaria: un estudio de casos. Revista de psicodidáctica, Vizcaya, v. 11, n. 2, p. 281-291, 2006.

GUERRINI Sebastian. Introducción a la Mirada. 2006. Disponible en: <http://www.guerriniisland.com/espaniol/escritos/ introduccion_a_la_mirada.html>. Acceso en: 20 sept. 2011.

GÚTIEZ, Pilar. La evolución de la atención a las necesidades educativas especiales. In: GENTO, Samuel (Coord.). Educación Especial. v.2. Madrid: Sanz y Torres, 2003.

HASTAD, Douglas; LACY, Alan. Measurement and evaluation in physical education and exercise science. Boston: Allyn and Bacon, 1998.

HERNÁNDEZ, Roberto; FERNÁNDEZ, Carlos; BAPTISTA, Pilar. Metodología de la investigación. 2.ed. México: McGraw Hill, 1998. 
ILLÁN, Nuria. Materiales para trabajar la autonomía y vida independiente de las personas con discapacidad psíquica. FEISD: Madrid, 2001.

INSTITUTO NACIONAL DE ESTADÍSTICA. Encuesta sobre Discapacidades, Deficiencias, y Estado de Salud. 1999. Disponible en: <http://www.ine.es/prodyser/pubweb/discapa/discapamenu.html>. Acceso en: 20 sept. 2011.

IZUZQUIZA, Dolores. Tratamiento educativo de necesidades educativas de tipo intelectual. In: GENTO, Samuel (Coord.). Educación Especial. v.2. Madrid: Sanz y Torres, 2003.

JASPAR, Jos; HEWSTONE, Miles. La teoría de la atribución. In: Moscovici, Serge (Comp.). Psicología Social II. Madrid: Paidós, 1985.

JIMÉNEZ, Antonio. Encuesta sobre Discapacidades, Deficiencias y Estado de Salud (1999). Breve análisis de los primeros resultados. 2001. Disponible en: <http://usuarios.discapnet.es/AJimenez/encuesta/minusval.html>. Acceso en: 20 sept. 2011.

LEYENS, Jaques Phillippe; CODOL, Jean Paul. Cognición Social. In: HEWSTONE, Miles; et al.. Introducción a la Psicología Social. Barcelona: Ariel, 1993.

MALHOTRA, Naresh. Pesquisa de marketing: uma orientação aplicada. Porto Alegre: Bookman, 2001.

MANOEL, Edison de Jesus; DE CARVALHO, Yara Maria. Pós-graduação na educação física brasileira: a atração (fatal) para a biodinâmica. Educação e Pesquisa, São Paulo, v. 37, n. 2, p. 389-406, 2011.

MOLINA, Jesús; ILLÁN, Nuria. Educar para la diversidad en la escuela actual. Una experiencia práctica de integración curricular. Sevilla: MAD, 2008.

MOLINA, Jesús; ILLÁN, Nuria. El proceso de integración efectiva de los alumnos con síndrome de Down en Educación Infantil: variables influyentes en dicho proceso. Revista Española de Pedagogía, Madrid, v. 248, p. 5-22, 2011.

MOYA, Miguel. Cognición Social. In: MORALES, José Francisco (Coord.). Psicología Social. Madrid: Mac Graw Hill, 1999.

PÉREZ, César. Métodos estadísticos avanzados con SPSS. Madrid: Thomson, 2005.

RIEF, Sandra; HEIMBURGE, Julie. Como ensinar todos os alunos na sala de aula inclusiva. v.1, Porto: Porto Editora, 2000.

RODRÍGUEZ, Gabriela. ¿Cómo ves? El aborto. México: Dirección General de Divulgación de la Ciencia (UNAM), 2004.

RODRÍGUEZ, Silvia. Percepción y creación de una ciudad. Granada: Universidad de Granada, 2003. Disponible en: <http:// www.ugr.es/ pwlac/G19_17Silvia_Lopez_Rodriguez.html>. Acceso en: 20 sept. 2011.

SPIJKER, Jeroen; PÉREZ, Julio. La ocupación laboral de los convivientes con afectados por discapacidades: un análisis multivariable. Revista Internacional de Sociología (RIS), Córdoba, v. 68, n. 2, p. 311-332, 2010.

STAINBACK, Susane; STAINBACK, William; EAST, Katheryn; SAPON-SHEVIN, Mara. A commentary on Inclusion and the development of a positive self-identity by people with disabilities. Exceptional Children, Arlington, v. 60, n. 6, p. 486-490, 1994.

STONE, Addison; MAY, Alison. The accuracy of academic self-perceptions in adolescents with learning disabilities. Journal of Learning Disabilities, California, v. 35, p. 370-383, 2002.

TALLIS, Jaime. (Comp). Sexualidad y discapacidad. Madrid: Miño y Dávila Editores, 2005.

TOLIMSON, Sally. A Sociology of Special Education. London: Routledge, 1982.

TORRI, Danielle; STAIMBACH, Beatriz; FERNANDEZ, Alexandre. Sacrifícios, sonhos, indústria cultural: retratos da educação do corpo no esporte escolar. Educação e Pesquisa, São Paulo, v. 33, n. 3, p. 499-512, 2007. 
TURNER, John. Introducción: El campo de la Psicología social. In: MORALES, Francisco (Coord.). Psicología social. Madrid: Mac Graw Hill, 1999.

VERDUGO, Miguel Ángel. Análisis de la definición de la discapacidad intelectual de la Asociación Americana sobre Retraso mental de 2002. 2002. Disponible en: <http://inico.usal.es/publicaciones/pdf/AAMR_2002.pdf>. Acceso en: 20 sept. 2011.

ZUZA, Francisco Javier. La persona con discapacidad grave. Desafíos y líneas de acción pastoral. Santander: SAL TERRAE, 2000.

Recebido en: 05.10.2011

Aprobado en: 10.05.2012

Jesús Molina Saorín es profesor en el Departamento de Didáctica y Organización Escolar de la Facultad de Educación de la Universidad de Murcia (España); profesor-tutor en la Universidad Nacional de Educación a Distancia (UNED); doctor europeo en Pedagogía; doctorando en Educación Física y Máster en síndrome de Down.

Rui Manuel Nunes Corredeira es profesor en el Departamento de Educación Física Adaptada de la Facultad del Deporte de la Universidad de Porto (Portugal); doctor en Educación Física y máster en Actividad Física Adaptada.

E-mail: rcorredeira@fade.up.pt

Mónica Vallejo Ruiz es profesora titular en el Departamento de Didáctica y Organización Escolar de la Facultad de Educación de la Universidad de Murcia (España); Doctora en Pedagogía. E-mail: monicavr@um.es 